Conclusion This study demonstrates that despite evidence of Vitamin D and its role in anti-inflammatory and immune-modulating effects (in addition to bone protection) we are not actively measuring and treating its deficiency. Perhaps guidance from ECCO may encourage our testing of vitamin D in IBD patients.

Competing interests None declared.

\section{PWE-248 DOCOSAHEXANOEIC ACID IN THE AETIOLOGY OF CROHN'S DISEASE-DATA FROM A EUROPEAN PROSPECTIVE COHORT STUDY (EPIC)}

doi:10.1136/gutjnl-2012-302514d.248

S Chan.* Department of Gastroenterology, Norfolk and Norwich University Hospitals NHS Foundation Trust, Norwich, UK

Introduction Docosahexaenoic acid (DHA) is an n-3 polyunsaturated fatty acid, present in fish oils, which possess anti-inflammatory properties, including biological effects on genetic expression, immune cell function and the production of inflammatory eicosonoids. The aim of this investigation was to conduct the first prospective cohort study to determine if low dietary intakes of this nutrient were associated with the development of incident Crohn's disease.

Methods A total of 229702 healthy participants aged 30-74 years of age were recruited in a prospective cohort study (EPIC-European Prospective Investigation Into Cancer) who were resident in either: Sweden, Denmark, The Netherlands, Germany or The UK. At baseline, participants completed food frequency questionnaires from which their intakes of DHA and other dietary fatty acids were determined. The cohort was followed-up between 1992 and 2004 to identify those who developed incident Crohn's disease, with each diagnosis medically confirmed by a review of the medical notes. Each case was matched with four controls for gender, age at recruitment and centre, and the analysis performed using conditional logistic regression. Adjustments were made for: smoking, total energy, fat and fatty acids, which affect the inflammatory process.

Results In the cohort, 73 participants developed incident Crohn's disease (64\% women) at a mean age of 56.3 years ( $\mathrm{SD}=11.1$ years). Of these, $51 \%$ had disease affecting the terminal ileum and $19 \%$ had a pancolitis. The four higher quintiles of DHA intake were all inversely associated with the development of Crohn's disease, with the highest (>310 mg/day) having the largest effect (OR 0.07, 95\% CI 0.01 to $0.80, p=0.03$ ), with a dose-response across categories (OR trend $0.54,95 \% \mathrm{CI} 0.30$ to $0.98, \mathrm{p}=0.04)$. The attributable fraction, namely the percentage of cases which may be due to the lowest dietary intake of DHA was $40 \%$.

Conclusion The data suggest a potential dose-dependent protective effect for increasing dietary DHA in the aetiology of Crohn's disease. DHA should be measured in future aetiological studies of this disease and could be assessed as a dietary treatment in clinical trials of patients.

Competing interests None declared.

\section{PWE-249 BODY MASS INDEX IN THE AETIOLOGY OF INFLAMMATORY BOWEL DISEASE-DATA FROM A EUROPEAN COHORT STUDY (EPIC)}

doi:10.1136/gutjnl-2012-302514d.249

S Chan.* Department of Gastroenterology, Norfolk and Norwich University Hospitals NHS Foundation Trust, Norwich, UK

Introduction There are plausible biological mechanisms for how obesity may be involved in the development of inflammatory bowel disease (IBD) due to the pro-inflammatory cytokines synthesised by adipose tissue including TNF- $\alpha$. The aim of this work was to conduct the first prospective cohort study of obesity in the aetiology of IBD.

Methods The cohort consisted of 366351 healthy men and women aged 30-74 years from centres in Europe (EPIC-European Prospective Investigation into Cancer) recruited between the years 1991 and 1998. At recruitment participants' weight, height and energy intake were measured and physical activity from questionnaires and their body mass index (BMI) calculated. The cohort was monitored to 2004 to identify those who developed Crohn's disease (CD) or ulcerative colitis (UC). Each diagnosis was medically confirmed via review of the medical notes. Each case was matched with four controls, for gender, age at recruitment and centre. BMI was divided into five categories. Conditional logistic regression was used to calculate OR and 95\% CI adjusted for smoking.

Results In the cohort, 102 participants developed incident CD (73\% female, mean age of 55.9 years, $\mathrm{SD}=11.0$ years) and 213 participants developed incident UC (61\% female, mean age of 57.5 years, $\mathrm{SD}=10.8$ years). There were no associations between the four higher categories of BMI and the development of CD (Trend $=1.00,95 \% \mathrm{CI}$ 0.79 to $1.27, \mathrm{p}=0.91$ ) or UC (Trend $=1.03,95 \%$ CI 0.83 to $1.29, \mathrm{p}$ 0.76). Dividing BMI in two categories.

Conclusion No associations were found between BMI and either CD or UC. Further studies are required to confirm these results. If confirmed then BMI does not need to be measured in future epidemiological studies of IBD.

Competing interests None declared.

\section{PWE-250 AZATHIOPRINE AND ALLOPURINOL CO-THERAPY FOR IBD PATIENTS IS A SAFE AND EFFECTIVE TREATMENT OPTION IN THE DISTRICT GENERAL HOSPITAL SETTING}

doi:10.1136/gutjnl-2012-302514d.250

H E Johnson, S A Weaver, S D McLaughlin. " Department of Gastroenterology, Royal Bournemouth Hospital, Bournemouth, UK

Introduction Reports from specialist inflammatory bowel disease (IBD) units have demonstrated that allopurinol and low-dose thiopurine co-therapy is an effective treatment option in patients who have failed standard dose therapy. There is little experience however from the district general hospital (DGH) setting. Co-therapy was introduced in our unit in September 2010.

Aim To evaluate the safety and therapeutic outcome of IBD patients treated with azathioprine and allopurinol co-therapy at our institution.

Methods A prospective database of all patients treated with allopurinol co-therapy is maintained at our institution. We reviewed the database entries and case notes of all patients. Data from patients who had been on allopurinol for $<3$ months were disregarded.

Results 25 patients were identified, five were excluded because of insufficient follow-up ( $<3$ months) and one patient was lost to follow-up. The median length of co-therapy was 9 (range 3-12) months. Diagnosis was ulcerative colitis (12), Crohn's disease (7), IBD-Unclassified (1). Indications for co-therapy were abnormal LFTs (3), drug side effects (8), high methylmercaptopurine (5), gout (2), therapeutic failure (2). 6-thioguanine nucleotide levels were measured where appropriate in patients before co-therapy, of these $50.0 \%$ were therapeutic. Following co-therapy 6-thioguanine nucleotide levels were therapeutic in $81.8 \%$ of patients. Co-therapy was effective and well tolerated in $13(68.4 \%)$. Two (10\%) patients developed side effects from allopurinol both had been treated with $200 \mathrm{mg}$ od, in one patient $(50 \%)$ symptoms resolved with dose reduction.

Conclusion We have previously published our long-term outcome data of IBD patients treated with thiopurines; $55 \%$ of these patients stopped thiopurine therapy due to therapeutic failure or side effects. The current data demonstrate that the majority of this refractory 
group can be rescued with co-therapy. These data demonstrate that co-therapy is a safe and effective treatment option in the DGH setting.

Competing interests None declared.

\section{PWE-251 DISTRICT GENERAL HOSPITAL EXPERIENCE OF OPTIMISING TREATMENT OUTCOME ON THIOPURINES BY CO-PRESCRIPTION OF ALLOPURINOL IN PATIENTS WITH INFLAMMATORY BOWEL DISEASE}

doi:10.1136/gutjnl-2012-302514d.251

S Cherian,* A Gera, V Saxena, A Loganayagam. Department of Gastroenterology, Queen Elizabeth Hospital NHS Trust, London, UK

Introduction Numerous patients, especially those with elevated thiopurine methyltransferase (TPMT) activity, selectively methylate thiopurine drugs, generating high levels of methylated metabolites and low thioguanine nucleotides. This pattern of metabolism is related to hepatotoxicity and non-response to therapy. Co-prescription of thiopurines (TP) (at 25\% of standard dose) with allopurinol (xanthine oxidase inhibitor) seems to avoid this problem, optimising both metabolite profile and clinical response. British experience on the use of this combination therapy (CT) remains limited. In this study we report a district general hospital (DGH) experience for the indications of toxicity (mainly hepatic) and very high TPMT activity in patients with inflammatory bowel disease (IBD).

Methods Retrospective notes review of patients at a district general hospital treated with CT using 25\% dose of TP and $100 \mathrm{mg}$ allopurinol was undertaken. Particular attention was paid to whether CT overcame the specific problem that prevented thiopurine monotherapy

Results 15 patients (age $24-77$ yrs, male $=6$, Crohn's=6, ulcerative colitis=9) were identified. All 15 patients were on an oral five amino salicylic acid preparation and 12 patients had previously been on a TP. Two patients with fibrotic stricture and one patient with hepatic steatosis were excluded from the analysis. Of those patients receiving co-prescription for side effects (four hepatoxicity and five others: rash, nausea, headache, fatigue), $78 \%$ were able to tolerate CT with complete resolution of liver function abnormality where relevant. Clinical remission was achieved in $100 \%$ of the patients who tolerated CT. In the three patients where CT was commenced for very high TPMT activity, 1 (33\%) developed non-specific side effects (headache, nausea) leading to discontinuation of therapy and $2(67 \%)$ achieved clinical remission.

Conclusion CT with low-dose TP and allopurinol avoids hepatotoxicity and improves chances for clinical remission. CT may also prevent other side effects. CT should be fully utilised in a DGH for hepatoxicity and other side effects. Using CT as first line in those with high TPMT activity remains questionable and requires further scrutiny in a prospective study.

Competing interests None declared.

\section{PWE-252 TRENDS IN IMAGING AND IMPACT OF DIAGNOSTIC MEDICAL RADIATION IN INFLAMMATORY BOWEL DISEASE}

doi:10.1136/gutjnl-2012-302514d.252

${ }^{1} \mathrm{~S}$ Chatu, ${ }^{*} \mathrm{~A}$ Poullis, ${ }^{1} \mathrm{R}$ Holmes, ${ }^{2} \mathrm{R}$ Greenhalgh, ${ }^{1} \mathrm{R}$ Pollok. ${ }^{1}$ Department of Gastroenterology, St George's Hospital, London, UK; ${ }^{2}$ Department of Radiology, St George's Hospital, London, UK

Introduction Increasing use of diagnostic imaging in inflammatory bowel disease has led to concerns about the malignant potential of ionising radiation in a cohort that is already predisposed to malignancy. The aim was to quantify radiation exposure in inflammatory bowel disease patients referred from primary care, determine predictors of high exposure and evaluate temporal changes in imaging at a single centre.

Methods Patients with a diagnosis of Crohn's disease (CD) or ulcerative colitis (UC) were prospectively recruited from clinic between January 2011 and June 2011. Demographic and clinical data were obtained by scrutinising medical records. The number and type of imaging procedures was obtained from the radiology database which was first set up in 1990, we only included those diagnosed after the database was initiated. The effective dose of radiation from each test was estimated from published standardised tables. Cumulative effective dose (CED) was calculated for each subject by summing the effective doses of radiation from diagnosis until end of study period which was June 2011. Cox regression analysis was performed to assess for factors associated with potentially harmful levels of ionising radiation defined as total CED $>50 \mathrm{mSv}$.

Results The cohort included 415 patients. Median disease duration for Crohn's disease and ulcerative colitis was 8.3 and 7.7 years. Median total CED was $7.2 \mathrm{mSv}$ (IOR 3.0-22.7) in Crohn's disease and $2.8 \mathrm{mSv}$ (IOR $0.8-8.9$ ) in ulcerative colitis patients. A total of 32 patients (8\%) received a CED $>50 \mathrm{mSv}$. Multivariate analysis revealed a history of IBD related surgery had a HR of 7.7. During the study period usage of abdominal CT increased by $350 \%$.

Conclusion About 1 in 10 patients were exposed to potentially harmful levels of ionising radiation therefore strategies to reduce radiation exposure are needed. While there was an increased uptake of both MRI and small bowel ultrasound over the past 20 years use of CT also increased substantially.

Competing interests None declared.

\section{PWE-253 ADALIMUMAB IMPROVES HEALTH-RELATED OUALITY OF LIFE FOR 52 WEEKS IN PATIENTS WITH ULCERATIVE COLITIS}

doi:10.1136/gutjnl-2012-302514d.253

${ }^{1} \mathrm{~W} J$ Sandborn, ${ }^{2} \mathrm{G}$ Van Assche, ${ }^{3} \mathrm{R}$ B Thakkar, ${ }^{4} \mathrm{~A}$ Lazar, ${ }^{4} \mathrm{M}$ Kron, ${ }^{3} \mathrm{M}$ Yang ${ }^{5} \mathrm{~S}$ P Patel, ${ }^{*} \mathrm{~J}$ Chao, ${ }^{3} \mathrm{P}$ M Mulani. ${ }^{1}$ UCSD, La Jolla, California, USA; ${ }^{2}$ U Hosp Gasthuisberg, Leuven, Belgium; ${ }^{3}$ Abbott, Abbott Park, Illinois, USA; ${ }^{4}$ Abbott, Ludwigshafen, Germany; ${ }^{5}$ Abbott, Maidenhead, UK

Introduction We investigated effects of adalimumab (ADA) maintenance therapy on health-related quality of life (HRQOL) through 52 weeks (wks) in patients (pts) with ulcerative colitis (UC).

Methods 494 pts with moderate to severe UC (Mayo score, 6-12 points; endoscopic subscore, 2-3 points; anti-tumour necrosis factor [anti-TNF]-naïve and anti-TNF-experienced [40.3\%]) who had failed conventional therapy were enrolled in a $52-w k$, randomised, double-blind, placebo ( $\mathrm{PBO}$ )-controlled maintenance trial. ADA-treated pts received induction therapy (160/80 mg@Wks 0/2) and 40-mg every-other-week (eow) maintenance therapy. Pts with inadequate response could switch to open-label eow therapy after Wk 12 and subsequently to weekly therapy. HROOL was measured by Inflammatory Bowel Disease Questionnaire (IBDQ). Intent-totreat population was analysed. IBDQ response rates were compared between treatment groups using Cochran-Mantel-Haenszel test stratified for prior anti-TNF use whereas $\chi^{2}$ test was used in antiTNF-naïve pts. Non-responder imputation was used for response variables. For change of IBDQ scores, ANCOVA model with treatment and prior anti-TNF status as factors and baseline value as covariate was used. Missing values were imputed through last observation carried forward (LOCF).

Results Significantly more ADA-treated pts were IBDQ responders (increase in IBDQ score $\geq 16$ points from baseline) throughout Wks 